\title{
Incidence and prevalence of idiopathic pulmonary fibrosis in US adults 18-64 years old
}

\author{
Ganesh Raghu', Shih-Yin Chen², Qiang Hou², Wei-Shi Yeh² and \\ Harold R. Collard ${ }^{3}$
}

Affiliations: ${ }^{1}$ Division of Pulmonary and Critical Care Medicine, Dept of Medicine, University of Washington, Seattle, WA, USA. ${ }^{2}$ Health Economics and Outcomes Research, Biogen, Cambridge, MA, USA. ${ }^{3}$ Division of Pulmonary and Critical Care Medicine, Dept of Medicine, University of California San Francisco, San Francisco, CA, USA.

Correspondence: Ganesh Raghu, University of Washington, Campus Box 356175, Seattle, WA 98195, USA. E-mail: graghuduw.edu

ABSTRACT We sought to present the epidemiology of idiopathic pulmonary fibrosis (IPF) in adults 18-64 years old in the USA.

From adults aged 18-64 years in a large administrative claims data in 2004-2010, patients with IPF were identified using diagnosis codes. We estimated annual incidence and cumulative prevalence of IPF over time, and examined potential risk factors for the IPF diagnosis.

The annual cumulative prevalence increased steadily in the first few years (from 13.4 cases per 100000 persons in 2005 to 18.2 cases in 2010 per 100000 persons), which is likely due to a methodological reason, while the annual incidence of IPF decreased over time (from 7.9 cases per 100000 person-years in 2005 to 5.8 cases in 2010 per 100000 person-years). The overall decrease was mainly driven by a decreasing trend in the younger patients (aged 18-44 years), while the incidence in older patients remained stable. Consistent trends were observed in subgroups defined by previously published more restrictive algorithms for diagnosis. Older age and male sex were associated with a higher incidence of disease $(\mathrm{p}<0.05)$.

In US adults younger than 65 years, we observed a decreasing incidence of IPF over time which may partially explain the plateau of cumulative prevalence in the last few years of our data.

@ERSpublications

Incidence of IPF decreased from 2004 to 2010 in US adults aged 18-64 and the cumulative prevalence reached a plateau http://ow.ly/YgpLD

This article has supplementary material available from erj.ersjournals.com

Received: Oct 062015 | Accepted after revision: Feb 082016 | First published online: April 282016

Support statement: This study was funded by Biogen. Aside from the funding, the company had no role in the study and no input or contributions into the findings thereof. Funding information for this article has been deposited with FundRef.

Conflict of interest: Disclosures can be found alongside this article at erj.ersjournals.com

Copyright @ERS 2016 


\section{Introduction}

Idiopathic pulmonary fibrosis (IPF), a specific form of chronic lung disease characterised by progressive scarring and fibrosis of the lungs, causes an irreversible loss of pulmonary function [1-3]. It is a highly morbid and ultimately fatal disease with a median survival estimated at 2-5 years from the time of diagnosis [4-9]. The mortality rate of IPF patients has been reported to be as high as 13.36 per 100000 (age-standardised rate in Northern Ireland) [10]. Most of the deaths among patients with IPF are due to respiratory failure or complicating comorbidity [11-13]. However, the accurate diagnosis of IPF can be challenging. While the 2011 evidence-based guidelines [3] provide criteria for identifying usual interstitial pneumonia patterns and stipulate that the diagnosis of IPF requires careful evaluation for alternative causes, there are no standardised and validated questionnaires that can be utilised to ascertain the exclusion of all factors known to cause pulmonary fibrosis. The accuracy of diagnosis is increased through multidisciplinary discussions by experienced experts at regional centres for interstitial lung disease, acknowledging that not all patients will experience such a multidisciplinary discussion approach.

The incidence of IPF varies but has increased over time in most countries worldwide [10]. This trend is consistent with the increasing disease awareness on the part of physicians, the growth of pulmonary medicine and the implementation of increasingly sensitive imaging techniques [14]. However, limited published data are available for the epidemiology of IPF in the USA, especially for the trend over time. Five studies were identified that examined the prevalence and incidence of IPF in the USA [5, 9, 15-17]; only one study demonstrated the trend over time using national data $[9,16]$. The study, based on a nationally representative sample of the Medicare population (65 years or older), showed that the annual incidence of IPF remained stable from 2001 to 2011, with an overall estimate of 93.7 cases per 100000 person-years during the study period; meanwhile, the prevalence rate increased steadily from 202.2 cases per 100000 persons in 2001 to 494.5 cases per 100000 persons in 2011 [9]. While the trends in incidence and prevalence of IPF have been updated in the US population aged over 65 years who are insured through the national Medicare programme [9], comparable epidemiology data for people younger than 65 years, a population most likely to be insured through commercial insurance plans, is unknown.

The purpose of this study was to update the epidemiology data of IPF among younger adults in the USA. Although IPF is known to occur predominantly in the elderly, its definition allows for a diagnosis to be made in patients as young as 18 years [3]. Thus, it is important to understand the epidemiology in adults less than 65 years of age in order to give a complete picture of the burden of this disease and understand the need for early recognition during the disease course for prompt treatment. This study estimated the trends of incidence and prevalence, and explored risk factors of IPF among adults aged 18-64 years from a large commercially insured population in the USA.

\section{Methods}

\section{Data source}

The retrospective administrative patient claims data used in this study were from Optum's Clinformatics ${ }^{\mathrm{TM}}$ Data Mart for MultiPlan Database. The dataset is from a national sample of more than 45 managed care health plans covering nine census regions with more than 89 million lives. The population is geographically diverse across US census regions. The database contains medical claims, pharmacy claims and associated costs, along with information on health plan enrolment and demographic characteristics. The medical and pharmacy claims files contain details on date of service, place of service, International Classification of Diseases, Ninth Revision, Clinical Modification (ICD-9-CM) diagnosis codes, Current Procedural Terminology codes, provider type, National Drug Codes and other detailed information regarding patients' outpatient prescriptions.

The data between January 1, 2004 and December 31, 2010 (study period) were analysed, and health plan enrolees who were 18-64 years old during the study period were included in the analyses.

\section{Patient identification}

The most recently published algorithm to identify patients with IPF from administrative claims data was used [9]. Specifically, candidate IPF patients were first identified if they had at least one claim with ICD-9-CM diagnosis code 516.3 (idiopathic fibrosing alveolitis, which is used by providers to identify IPF) during the study period. Prevalent IPF patients were confirmed if they did not have a diagnosis code for any other interstitial lung diseases (online supplementary table S1, except for the diagnosis code 515 for post-inflammatory pulmonary fibrosis, which is a commonly used general diagnostic code for IPF) after the last claim with the diagnosis code 516.3. Patients with incident IPF were required to have at least 1 year of continuous enrolment in medical and pharmacy plans before the first diagnosis code 516.3. These patients were our primary cohort. 
Two subgroups of the primary cohort were further identified by applying additional criteria to form more restrictive cohorts of prevalent IPF patients, as investigated in the previous studies $[9,16]$. The first subgroup (broad case definition) excluded patients if they had a claim with the diagnosis code 515 after the last diagnosis code 516.3. The second subgroup (narrow case definition) further restricted the broad case subgroup patients by requiring a claim for a surgical lung biopsy, transbronchial lung biopsy or computed tomography scan of the thorax prior to the last diagnosis code 516.3 .

\section{Data analysis}

Summary statistics of demographic variables (age at diagnosis, sex and census region) were reported for incident IPF patients identified in the primary cohort and subgroups, respectively. The demographics of health plan enrolees in 2010, aged 18-64 years, were also described to provide an overview of the study population. The prevalence of selected comorbidities of interest (online supplementary table S2) during the 1 year before the incident IPF diagnosis was also reported, defined as the percentage of patients with the corresponding ICD-9-CM diagnosis codes on the medical claims.

The annual cumulative prevalence and annual incidence of IPF in each calendar year from 2005 to 2010 were calculated. We did not calculate these for 2004 because we did not have prior data to ascertain incident cases. To calculate the cumulative prevalence, patients were regarded as prevalent cases from the date of IPF diagnosis and onwards. Incidence rate was calculated by dividing the number of new cases by person-years at risk and presented as the number of cases per 100000 person-years. The annual incidence rate was further stratified by age group. The analyses on the annual prevalence and incidence rates were also conducted among the subgroups of the primary cohort.

Overall incidence of IPF between 2005 and 2010 was calculated, and then reported by demographic variables. A Poisson regression, with age group, sex and census region as explanatory variables, was estimated to assess the association between these demographic variables and the incidence of IPF. Incidence rate ratio $(95 \% \mathrm{CI})$ was reported.

\section{Results}

The 2004-2010 Optum Clinformatics ${ }^{\mathrm{TM}}$ data contain more than 54 million enrolees, including more than 40 million aged 18-64 years (figure 1). A total of 12852 patients had at least one claim with the diagnosis code 516.3 in the study period (candidate IPF patients). After further exclusion of other interstitial lung diseases, there were 7671 prevalent IPF patients identified, including 4206 incident cases (primary cohort). The broad case subgroup included 2670 patients and the narrow case subgroup included 1685 patients. The incident IPF patients (primary cohort) of this commercially insured population had a mean age of 53 years, $50.6 \%$ were female and they were geographically diverse across US census regions (table 1). Compared with the general young adults who enrolled in Optum health plans in 2010, the incident IPF patients were at least 10 years older but had similar sex and region distribution. The demographics of the two subgroups of the incident patients were similar to those in the primary cohort.

The occurrence of comorbid pulmonary conditions was common during the 1 year before diagnosis of IPF (table 2), including chronic obstructive pulmonary disease $(28.7 \%)$, pneumonia $(21.4 \%)$, acute bronchitis and bronchiolitis (18.8\%), and asthma (18.6\%). The prevalence was also high (occurring in $>10 \%$ of the patients) for diabetes, fatigue, gastro-oesophageal reflux disease, coronary artery disease and depression. The prevalence of comorbidities before IPF diagnosis in the subgroups was similar to that in the primary cohort.

In this commercially insured population of those aged 18-64 years, the annual cumulative prevalence of IPF increased during the first few years in the study period, from 13.4 per 100000 persons in 2005 to 16.9 per 100000 persons in 2007, but reached a plateau in the last few years with a prevalence around 18 per 100000 persons from 2008 to 2010 (figure 2). The annual incidence of IPF decreased steadily over the years, ranging from 7.9 new cases per 100000 person-years in 2005 to 5.8 new cases per 100000 person-years in 2010. When stratified by age group (figure 3), the decreasing trend of the annual incidence rate was noticeable in the younger group (18-44 years) but was less obvious among older adults (45-54 years). The annual incidence rate remained relatively stable over time among adults older than 54 years but younger than 65 years.

The IPF prevalence and incidence in the subgroups were lower than those in the primary cohort (figure 2), due to the more restrictive case definitions. For the broad case subgroup, the annual incidence decreased from 5.1 to 3.6 new cases per 100000 person-years and the prevalence ranged from 8.4 to 11.3 per 100000 persons between 2005 and 2010. For the narrow case subgroup, the annual incidence decreased from 2.9 to 2.4 new cases per 100000 person-years and the annual prevalence ranged from 4.6 to 6.7 per 100000 


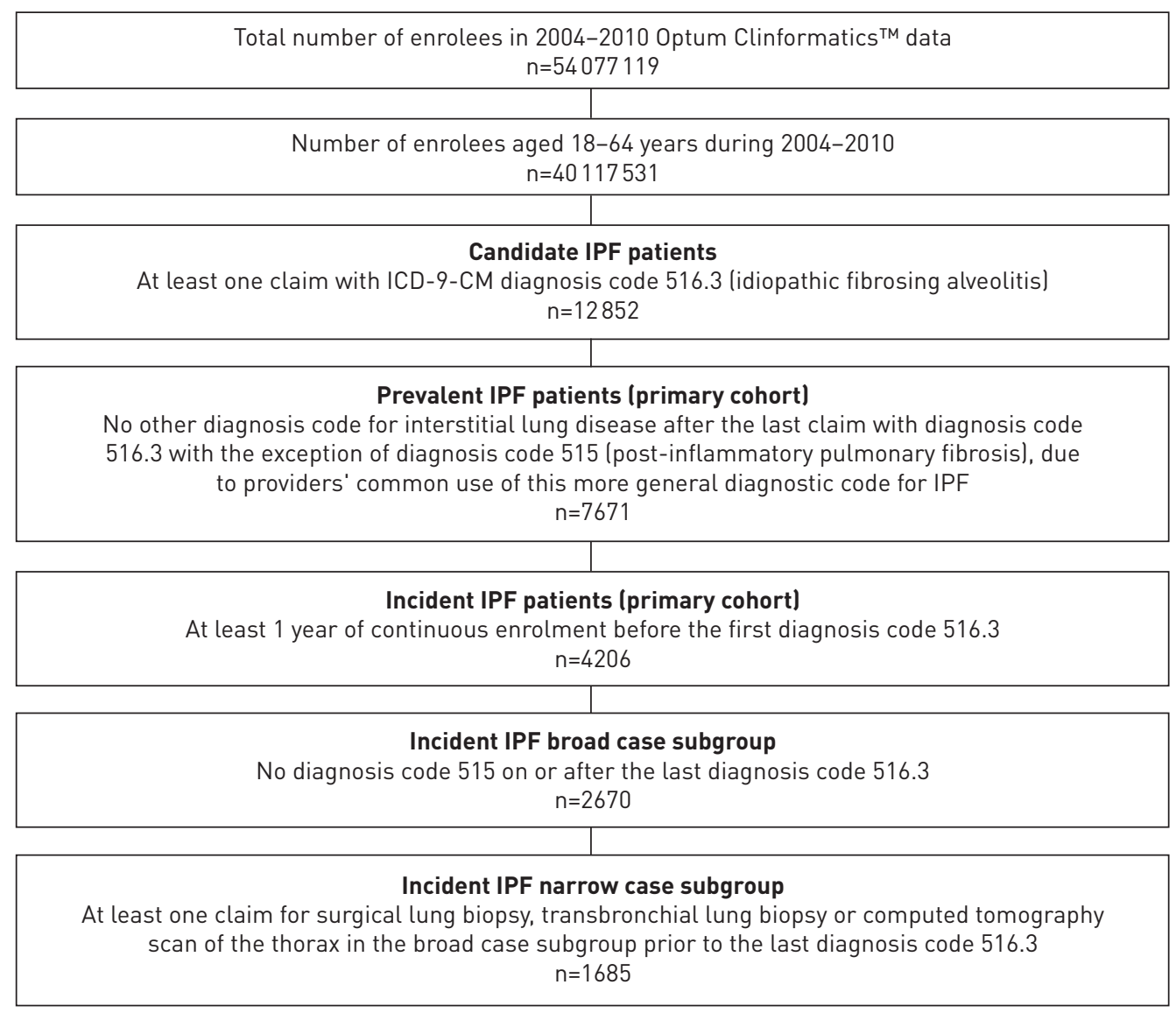

FIGURE 1 Sample selection. IPF: idiopathic pulmonary fibrosis; ICD-9-CM: International Classification of Diseases, Ninth Revision, Clinical Modification.

TABLE 1 Demographics of enrolees in 2010 and incident patients with idiopathic pulmonary fibrosis (IPF), overall and by subgroup

\begin{tabular}{|c|c|c|c|c|}
\hline & \multirow{2}{*}{$\begin{array}{l}\text { Optum } \\
\text { enrolees in } \\
2010\end{array}$} & \multicolumn{3}{|c|}{ IPF cohort } \\
\hline & & $\begin{array}{l}\text { Primary cohort } \\
\text { incident patients }\end{array}$ & $\begin{array}{l}\text { Broad case } \\
\text { subgroup }\end{array}$ & $\begin{array}{c}\text { Narrow case } \\
\text { subgroup }\end{array}$ \\
\hline Subjects $\mathrm{n}$ & 9375020 & 4206 & 2670 & 1685 \\
\hline Age (mean) at diagnosis years & 41.7 & 53.4 & 52.7 & 53.6 \\
\hline \multicolumn{5}{|l|}{ Age at diagnosis years } \\
\hline $18-34$ & 31.8 & 5.3 & 6.3 & 4.7 \\
\hline $35-44$ & 23.1 & 11.6 & 13.0 & 12.1 \\
\hline $45-54$ & 25.4 & 26.7 & 27.0 & 25.6 \\
\hline $55-64$ & 19.8 & 56.4 & 53.7 & 57.6 \\
\hline Female & 51.6 & 50.6 & 51.7 & 52.7 \\
\hline \multicolumn{5}{|l|}{ Census region } \\
\hline New England & 17.9 & 18.2 & 16.9 & 17.6 \\
\hline Middle Atlantic & 13.7 & 15.5 & 17.9 & 18.2 \\
\hline South Atlantic & 20.7 & 23.1 & 23.9 & 24.7 \\
\hline East South Central & 2.7 & 3.1 & 3.3 & 3.6 \\
\hline West South Central & 14.9 & 15.9 & 15.7 & 14.1 \\
\hline East North Central & 11.0 & 10.3 & 9.1 & 9.7 \\
\hline West North Central & 8.0 & 5.0 & 4.6 & 4.3 \\
\hline Mountain & 6.0 & 5.4 & 5.2 & 4.6 \\
\hline Pacific & 5.2 & 3.3 & 3.5 & 3.2 \\
\hline Other/unknown & 0.0 & 0.2 & 0.2 & 0.1 \\
\hline
\end{tabular}

Data are presented as \%, unless otherwise stated. 
TABLE 2 Prevalence of comorbidities during the 1 year before idiopathic pulmonary fibrosis (IPF) diagnosis

\begin{tabular}{|c|c|c|c|}
\hline & \multicolumn{3}{|c|}{ IPF cohort } \\
\hline & $\begin{array}{l}\text { Primary cohort } \\
\text { incident patients }\end{array}$ & $\begin{array}{l}\text { Broad case } \\
\text { subgroup }\end{array}$ & $\begin{array}{l}\text { Narrow case } \\
\text { subgroup }\end{array}$ \\
\hline Subjects n & 4206 & 2670 & 1685 \\
\hline \multicolumn{4}{|l|}{ Pulmonary and respiratory diseases } \\
\hline Chronic obstructive pulmonary disease & 28.7 & 26.1 & 30.7 \\
\hline Pneumonia & 21.4 & 18.9 & 24.6 \\
\hline Acute bronchitis and bronchiolitis & 18.8 & 18.1 & 19.8 \\
\hline Asthma & 18.6 & 18.4 & 20.0 \\
\hline Sleep apnoea & 8.5 & 7.8 & 8.6 \\
\hline Pulmonary embolism & 2.8 & 2.7 & 3.4 \\
\hline Pulmonary hypertension & 2.7 & 2.4 & 3.2 \\
\hline Lung cancer/bronchogenic carcinoma & 2.7 & 2.7 & 4.1 \\
\hline Influenza & 1.3 & 1.3 & 1.6 \\
\hline \multicolumn{4}{|l|}{ Cardiovascular diseases } \\
\hline $\begin{array}{l}\text { Coronary artery disease (exclusive of } \\
\text { myocardial infarction) }\end{array}$ & 15.7 & 14.5 & 16.4 \\
\hline Heart failure & 9.4 & 7.9 & 9.1 \\
\hline Cerebrovascular disease & 5.6 & 5.5 & 6.0 \\
\hline Atrial fibrillation & 4.2 & 3.9 & 4.1 \\
\hline Myocardial infarction & 2.8 & 2.8 & 2.9 \\
\hline Acute coronary syndrome & 2.2 & 2.4 & 2.4 \\
\hline Deep vein thrombosis & 1.1 & 0.9 & 1.4 \\
\hline \multicolumn{4}{|l|}{ Other diseases } \\
\hline Diabetes & 18.2 & 17.6 & 18.0 \\
\hline Fatigue & 17.5 & 17.9 & 19.6 \\
\hline Gastro-oesophageal reflux disease & 16.9 & 16.5 & 18.5 \\
\hline Depression & 10.2 & 10.4 & 11.3 \\
\hline Obesity & 5.3 & 5.2 & 4.8 \\
\hline Rib fractures & 0.6 & 0.4 & 0.7 \\
\hline
\end{tabular}

Data are presented as $\%$, unless otherwise stated.

persons between 2005 and 2010. The trends in these subgroups over the years were similar to the primary cohort (figure 2), including the trends across age groups (figure 3).

The overall incidence of IPF between 2005 and 2010 was 6.1 new cases per 100000 person-years (table 3). The incidence increased with age, ranging from 1.1 new cases per 100000 person-years among persons 18-34 years old to 19.3 new cases per 100000 person-years among persons 55-64 years old. With adjustment for sex and region, the incidence among subjects younger than 54 years old was $64 \%$ to $94 \%$
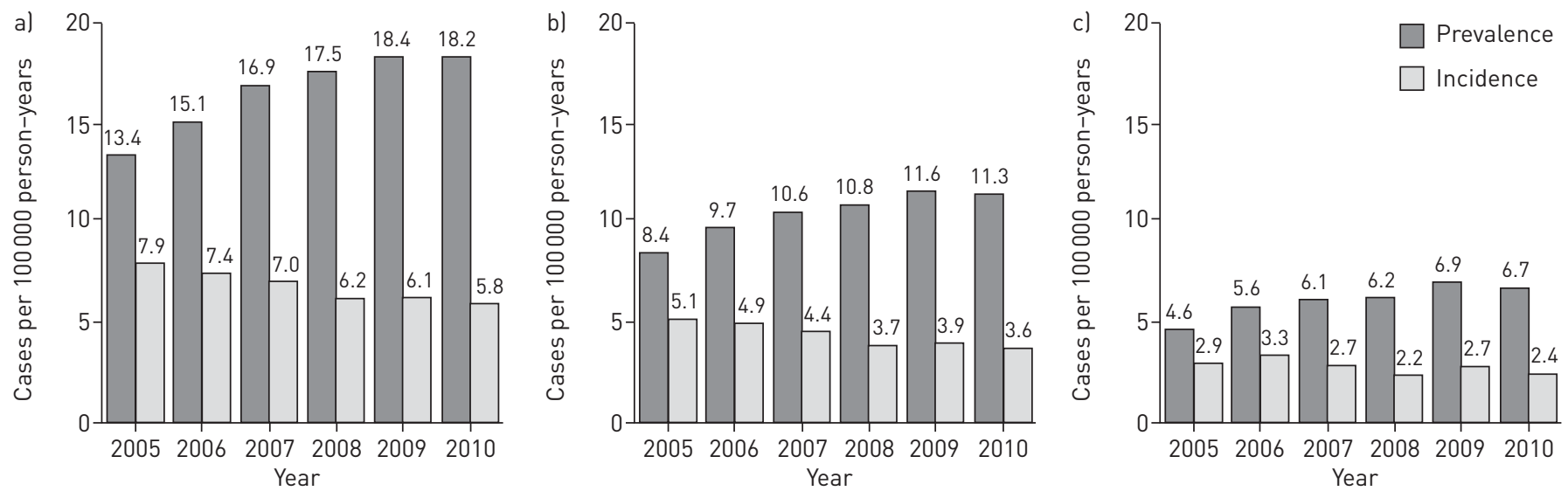

FIGURE 2 Annual prevalence and incidence rate of idiopathic pulmonary fibrosis in the commercially insured population aged 18-64 years, overall and by subgroup, 2005-2010: al primary cohort, b) broad case subgroup and c) narrow case subgroup. 

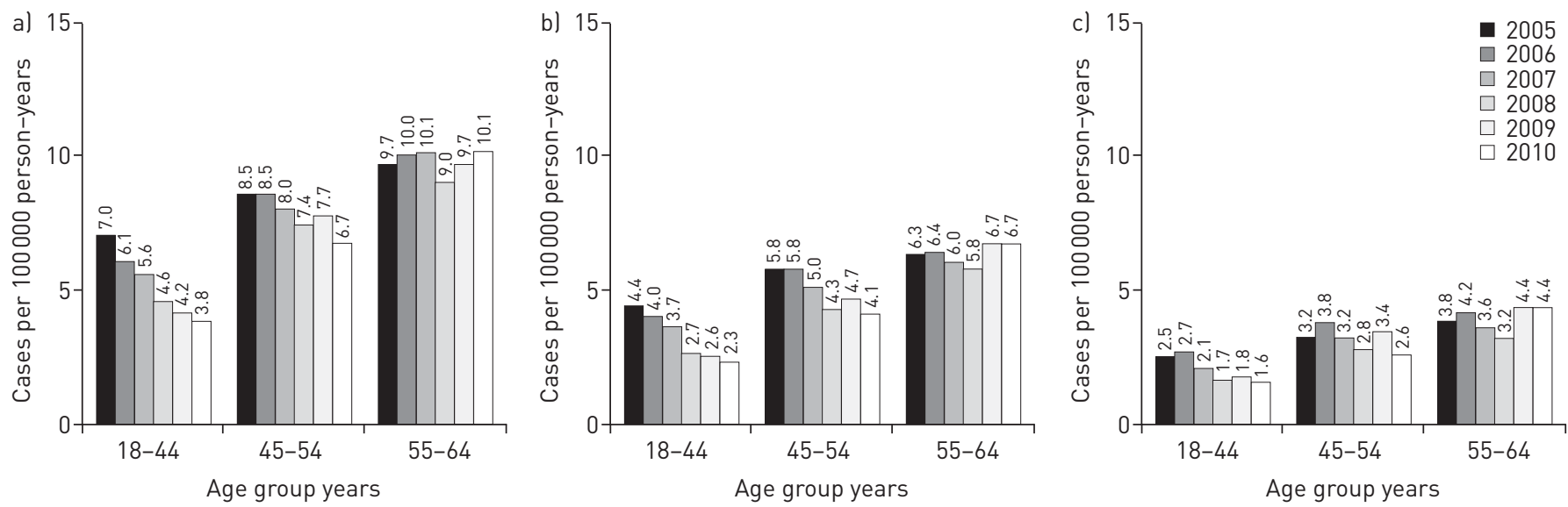

FIGURE 3 Annual incidence rate of idiopathic pulmonary fibrosis in the commercially insured population aged 18-64 years across age groups, overall and by subgroup, 2005-2010: a) primary cohort, b) broad case subgroup and c) narrow case subgroup.

lower (adjusted incidence rate ratio ranging from 0.06 for 18-34 years old to 0.36 for 45-54 years old, all $\mathrm{p}<0.05)$ than among the near elderly population (55-64 years old). Males had slightly higher incidence than females (6.4 versus 5.9 per 100000 person-years), and the difference was statistically significant after adjusting for age and region (adjusted incidence rate ratio 1.08, $\mathrm{p}<0.05$ ). Geographical variation in the incidence of IPF was also observed.

\section{Discussion}

Among adults aged 18-64 years from a commercially insured population, this study found that the annual incidence of IPF decreased over time from 2005 to 2010, while the annual cumulative prevalence of IPF increased steadily in the first few years. Age, sex and regions were independent predictors of the incidence rate of IPF in this population.

This study complements a recent study that reported incidence and prevalence of IPF among older Americans in the Medicare programme [9]. In the Medicare population, annual incidence of IPF remained stable between 2005 and 2010, and the cumulative prevalence increased over time. Contrary to the stable

TABLE 3 Factors associated with incidence of idiopathic pulmonary fibrosis (primary cohort)

Incidence rate ${ }^{\#}\left(95 \% \mathrm{CI}^{\text {ๆ }}\right.$

Adjusted incidence rate ratio $(95 \% \mathrm{CI})$

\begin{tabular}{lcc}
\hline $\begin{array}{l}\text { All enrolees } \\
\text { Age years }\end{array}$ & $6.1(5.9-6.4)$ & \\
$18-34$ & $1.1(1.0-1.3)$ & $0.06^{\S}(0.05-0.06)$ \\
$35-44$ & $2.6(2.4-2.9)$ & $0.15^{\S}(0.14-0.16)$ \\
$45-54$ & $6.4(6.0-6.9)$ & $0.36^{\S}(0.34-0.39)$ \\
$55-64$ & $19.3(18.4-20.3)$ & 1.00 (Reference) \\
Sex & & \\
Male & $6.4(6.1-6.7)$ & $1.08(1.02-1.15)$ \\
Female & $5.9(5.6-6.2)$ & 1.00 (Reference) \\
Census region & & \\
New England & $5.4(5.0-5.9)$ & $0.80^{\S}(0.71-0.90)$ \\
Middle Atlantic & $6.3(5.7-6.9)$ & $1.00(0.89-1.12)$ \\
South Atlantic & $7.9(7.3-8.5)$ & $1.22^{\S}(1.09-1.37)$ \\
East South Central & $8.2(6.7-9.8)$ & $1.07(0.88-1.30)$ \\
West South Central & $6.1(5.5-6.7)$ & $1.22^{\S}(1.08-1.37)$ \\
East North Central & $6.6(5.9-7.3)$ & $1.00($ Reference) \\
West North Central & $4.5(3.8-5.2)$ & $0.74^{\S}(0.63-0.87)$ \\
Mountain & $5.4(4.6-6.2)$ & $0.79 \S(0.68-0.93)$ \\
Pacific & $4.0(3.2-4.9)$ & $0.91(0.76-1.09)$ \\
Other/unknown & $9.1(2.8-25.2)$ & $1.72(0.64-4.61)$
\end{tabular}

\footnotetext{
\#: incidence rate reported as number of new cases per 100000 person-years using data from 2005 to 2010;
}

$\eta^{\text {: estimated based on Poisson distribution; }}{ }^{+}$: age at entry into the database; ${ }^{\S}$ : statistically significant at $p<0.05$. 
incidence observed in the older adults, we found a decreasing trend of IPF incidence among adults aged 18-64 years. When we stratified the analysis into more granular age subgroups, results showed that the decreasing trend of IPF incidence was mainly driven by the youngest group (18-44 years). This may be indicative of an increased awareness of disease definition which leads to a more prompt and accurate diagnosis of IPF, especially in younger adults whose interstitial lung diseases are commonly misdiagnosed as IPF. However, the trend for the annual incidence rate among the near-elderly population (55-64 years old) was the same as the older population aged over 65 years, which remained stable over time [9].

The initial increase of cumulative prevalence observed in this study is likely due to a methodological reason; methodologically, the cumulative prevalence estimates for the first few years are incomplete because the prevalent cases that were incident in the year before 2005 are not captured. This is supported by the fact that the cumulative prevalence reached a plateau. The plateau of cumulative prevalence of IPF in the last few years of the study period may also be partially explained by the decreasing incidence.

Our study assessed the demographic factors associated with IPF diagnosis, including age, sex and region. Race/ethnic origin was considered in the study on the Medicare population [9], but was not included in the current study due to absence of such data in the administrative claims database. As in the Medicare population, age was strongly associated with IPF incidence. The incidence differences between males and females were much smaller in this younger population in our study than in the Medicare population [9]. The differential in the association between sex and incidence of IPF by age groups was also observed in a previous study [16]. This pattern may be due to some modifying effect between age and sex, where the risk of IPF associated with male sex increases as people age. Consistent with the prior study in older adults [9], we also found the incidence of IPF to be generally higher in the southern regions (South Atlantic and West South Central) than the other regions of the USA.

The last report of the epidemiology of IPF among younger adults in the USA was published in 2006. That study used a nationwide healthcare claims dataset, and estimated the prevalence and incidence of IPF in 2000 using broad case and narrow case definitions, the same as the subgroups in our study, for males and females, respectively, in various age groups [16]. They reported that the incidence in males ranged from 2.8 per 100000 persons in the age group 18-34 years to 35.1 per 100000 persons in the age group 5564 years, based on the broad case definition of IPF; and ranged from 0.9 per 100000 persons in the age group 18-34 years to 12.2 per 100000 persons in the age group 55-64 years, based on the narrow case definition. The incidence rates among females were generally lower than males. The previously estimated incidence rates of IPF in 2000 were higher than the corresponding rates from 2005 to 2010 estimated in the subgroups in our study, which is consistent with the decreasing trend of IPF among younger adults observed in our study.

Our study reveals the following: 1) we updated the estimates of the epidemiology of IPF among young adults in the USA from recent national data, 2) we examined the incidence of IPF over time in greater detail than previously published and 3 ) our study is the first to report that the trend in incidence differs by age in adults younger than 65 years of age.

Our study also reported the prevalence of comorbidities in incident IPF patients aged 18-64 years. We found pulmonary and respiratory diseases to be the most common conditions before IPF diagnosis, consistent with other prior reports in the USA [18-20]. However, the high prevalence of pulmonary-related comorbidities before the IPF diagnosis could also be related to misdiagnosis. Fatigue, gastro-oesophageal reflux disease, depression, diabetes and coronary artery disease are common comorbid conditions, highlighting the need for multidisciplinary management in this patient group.

Our results should be interpreted with caution considering the following limitations inherent in such epidemiological studies. First, the case definition used to identify patients with IPF in this study was not validated with respect to medical charts and it is unknown if the diagnoses were based on multidisciplinary discussions. Without correction for false positives, these algorithms may overestimate the incidence and prevalence of IPF by as much as 50\% [17]. However, the negative predictive value of the algorithms is unknown and it is possible that many true IPF patients were not identified (i.e. false negatives) via those algorithms. In addition, the diagnostic coding system puts more barriers to evaluating the disease burden of IPF, as neither the ICD-9-CM system currently used in the USA nor the newer ICD-10-CM system have diagnosis codes specific to IPF. Nevertheless, the trends over years and/or across age groups observed in this study are robust since the findings were similar regardless of the algorithm for IPF diagnosis used. Next, our findings on a cohort of a commercially insured subgroup may not be applicable to populations aged 18-64 years who are covered by other insurance, such as Medicaid, or self-insured. Finally, data from commercial insurance plans have a relatively shorter follow-up due to employment turnover and lack of survival information, which limits our ability to further provide clinical explanation of the findings. 
In conclusion, the prevalence of IPF reached a plateau in the last few years of our study period among adults younger than 65 years in the USA. This may be partially explained by the decreasing incidence of IPF over time in this population due to more accurate diagnosis of IPF, especially in younger adults aged $18-44$ years.

\section{Acknowledgements}

The authors wish to acknowledge and thank Qian Li, Shuo Yang and Janet Dooley for their technical writing contributions to this manuscript. They are employees of Evidera (Bethesda, MD, USA), which provides consulting and other research services to pharmaceutical, device, government and nongovernment organisations. In their salaried positions, they work with a variety of companies and organisations, and are precluded from receiving payment or honoraria directly from these organisations for services rendered. Biogen paid Evidera for the work performed by the Evidera employees named above.

\section{References}

$1 \quad$ Meltzer EB, Noble PW. Idiopathic pulmonary fibrosis. Orphanet J Rare Dis 2008; 3: 8.

2 Michaelson JE, Aguayo SM, Roman J. Idiopathic pulmonary fibrosis: a practical approach for diagnosis and management. Chest 2000; 118: 788-794.

3 Raghu G, Collard HR, Egan JJ, et al. An official ATS/ERS/JRS/ALAT statement: idiopathic pulmonary fibrosis: evidence-based guidelines for diagnosis and management. Am J Respir Crit Care Med 2011; 183: 788-824.

4 Collard HR, King TE Jr, Bartelson BB, et al. Changes in clinical and physiologic variables predict survival in idiopathic pulmonary fibrosis. Am J Respir Crit Care Med 2003; 168: 538-542.

5 Fernandez Perez ER, Daniels CE, Schroeder DR, et al. Incidence, prevalence, and clinical course of idiopathic pulmonary fibrosis: a population-based study. Chest 2010; 137: 129-137.

6 King TE Jr, Tooze JA, Schwarz MI, et al. Predicting survival in idiopathic pulmonary fibrosis: scoring system and survival model. Am J Respir Crit Care Med 2001; 164: 1171-1181.

7 Latsi PI, du Bois RM, Nicholson AG, et al. Fibrotic idiopathic interstitial pneumonia: the prognostic value of longitudinal functional trends. Am J Respir Crit Care Med 2003; 168: 531-537.

8 Ley B, Collard HR, King TE Jr. Clinical course and prediction of survival in idiopathic pulmonary fibrosis. Am J Respir Crit Care Med 2011; 183: 431-440.

9 Raghu G, Chen SY, Yeh WS, et al. Idiopathic pulmonary fibrosis in US Medicare beneficiaries aged 65 years and older: incidence, prevalence, and survival, 2001-11. Lancet Respir Med 2014; 2: 566-572.

10 Hutchinson J, Fogarty A, Hubbard R, et al. Global incidence and mortality of idiopathic pulmonary fibrosis: a systematic review. Eur Respir J 2015; 46: 795-806.

11 King TE Jr, Pardo A, Selman M. Idiopathic pulmonary fibrosis. Lancet 2011; 378: 1949-1961.

12 Martinez FJ, Safrin S, Weycker D, et al. The cliical course of patients with idiopathic pulmonary fibrosis. Ann Intern Med 2005; 142: 963-967.

13 King TE Jr, Albera C, Bradford WZ, et al. All-cause mortality rate in patients with idiopathic pulmonary fibrosis. Implications for the design and execution of clinical trials. Am J Respir Crit Care Med 2014; 189: 825-831.

14 Samet JM, Coultas D, Raghu G. Idiopathic pulmonary fibrosis: tracking the true occurrence is challenging. Eur Respir J 2015; 46: 604-606.

15 Coultas DB, Zumwalt RE, Black WC, et al. The epidemiology of interstitial lung diseases. Am J Respir Crit Care Med 1994; 150: 967-972.

16 Raghu G, Weycker D, Edelsberg J, et al. Incidence and prevalence of idiopathic pulmonary fibrosis. Am J Respir Crit Care Med 2006; 174: 810-816.

17 Esposito DB, Lanes S, Donneyong M, et al. Idiopathic pulmonary fibrosis in US automated claims: incidence, prevalence and algorithm validation. Am J Respir Crit Care Med 2015; 192: 1200-1207.

18 Collard HR, Chen SY, Yeh WS, et al. Health care utilization and costs of idiopathic pulmonary fibrosis in U.S. Medicare beneficiaries aged 65 years and older. Ann Am Thorac Soc 2015; 12: 981-987.

19 Collard HR, Ward AJ, Lanes S, et al. Burden of illness in idiopathic pulmonary fibrosis. J Med Econ 2012; 15: 829-835.

20 Wu N, Yu YF, Chuang CC, et al. Healthcare resource utilization among patients diagnosed with idiopathic pulmonary fibrosis in the United States. J Med Econ 2015; 18: 249-257. 